\title{
Quantitative Measurement of Liver Function: The Quest for the Holy Grail?
}

$\mathbf{T}$ he quantitative measurement of liver function remains a Holy Grail of medicine. The absence of a reliable and readily available function test for the liver is in sharp contrast to organs such as the kidney ( 1 ) and lung (2), for which such tests exist and are used in a wide variety of applications including prognostication and determination of the need for solid organ transplantation.

It is easy to see how such a test would have wide applicability in clinical practice. It would provide valuable prognostic information in the setting of cirrhosis, for which a histologic diagnosis that offers no information about liver function is often used as a surrogate to indicate

\section{See page 294}

impaired liver function. Quantitative measurement of liver function would be of tremendous value in assessing the quality of donor organs and suitability of recipients in the setting of liver transplantation. It would also provide a more scientific assessment of surgical risk and the likelihood of decompensation after hepatectomy in a variety of clinical scenarios, including resection of the increasingly prevalent hepatocellular carcinoma and after live donor liver transplantation.

With this in mind, de Graaf et al. (3) published their results in this edition of The Journal of Nuclear Medicine, comparing the performance of various

Received Jul. 29, 2010; revision accepted Aug. 11, 2010.

For correspondence or reprints contact: Pierre M. Gholam, University Hospitals Case Medical Center, 11100 Euclid Ave., WRN5066, Cleveland, $\mathrm{OH} 44106$.

E-mail: pierre.gholam@case.edu

COPYRIGHT (C) 2011 by the Society of Nuclear

Medicine, Inc.

DOI: 10.2967/jnumed.110.080515 tests of liver function in a $70 \%$ partial hepatectomy rat model. The authors aimed to compare 2 nuclear imaging techniques, namely ${ }^{99 \mathrm{~m}} \mathrm{Tc}$-galactosyl human serum albumin (99mTc-GSA) scintigraphy and ${ }^{99 \mathrm{~m}} \mathrm{Tc}-(2,4,6$-trimethyl3-bromo)-iminodiacetic acid $\left({ }^{99 \mathrm{~m} T c}\right.$ mebrofenin) hepatobiliary scintigraphy (HBS), as well as 2 dynamic liver function tests-the indocyanine green (ICG) clearance test and the galactose elimination capacity (GEC) - in the assessment of hepatic function during liver regeneration. Data were compared against a widely clinically used standard: the postoperative increase in remnant liver volume using CT volumetry and the percentage of proliferating hepatocytes. The results suggest a limited value of GEC, which appears to be affected by factors other than liver regeneration at all time points after hepatectomy. In contrast, ${ }^{99 \mathrm{~m} T c-G S A}$ uptake appears to underestimate hepatic regeneration in comparison to liver volume and ${ }^{99 \mathrm{~m}} \mathrm{Tc}-\mathrm{meb}$ rofenin uptake. de Graaf et al. (3) conclude that ${ }^{99 \mathrm{~m} T c-}$ mebrofenin HBS may be the most advantageous test because it appears to provide visual and quantitative information on liver function.

The study by de Graaf et al. (3) addressed issues regarding the evaluation of recovery of liver function that had long been pondered but not fully investigated. The intriguing results certainly highlight the discrepancy between different tests that has clouded the field for many years. Although the lack of fluctuation in liver function after hepatectomy when measured by GEC probably highlights its limitations in reflecting changes after hepatectomy (Table 3 in de Graaf et al. (3)), this result may be because GEC is unlikely to change-even with significant impairment of liver volume- because of the ability of the remaining healthy liver to effectively phosphorylate galactose. These data, if confirmed, could alter the ongoing effort to develop ${ }^{18} \mathrm{~F}$-labeled fluorogalactose for the measurement of GEC with PET (4). However, it will be more interesting to see if these results will change in the setting of preexisting hepatic fibrosis, which can be induced experimentally. The underestimation of liver generation by 99mTc-GSA uptake may also reflect a component of liver function that may take longer to recover after hepatectomy, suggesting that ${ }^{99 \mathrm{~m}} \mathrm{Tc}-\mathrm{GSA}$ uptake may indeed be of value in tracking liver function recovery more chronically. This possibility is suggested by the slow but sustained increase of liver function by day 7 after hepatectomy (Table 3 in de Graaf et al. (3)).

An interesting and previously reported finding is the development of mild to moderate steatosis after hepatectomy (5). Although the exact determinants and implications of steatosis in this setting are not clear, previous studies have suggested a possible deleterious effect on indices of hepatic regeneration (6). Whether the effect of steatosis differentially influences the performance of the tests used is unclear and would probably require a quantitative or semiquantitative assessment of triglyceride content in the liver-an assessment that would certainly be worth performing in the future.

In contrast to previous experiments using similar models (7), rats in the current study failed to return to their baseline liver volume. Whether the presence of steatosis, the experimental setup of overnight fasting, or other factors may have affected regeneration is fraught with controversy because fasting had been previously reported to favorably affect regeneration (8). Nonetheless, it 
is certainly worth further exploration. Despite this impairment in volumetric regeneration, functional recovery appears to have been achieved. The causes for this discrepancy may indeed reflect the pitfalls of assessments of liver recovery after hepatectomy based purely on volume measurement. Conversely, the causes may indicate the limitations of using a single test for the assessment of function in an organ that performs an extremely wide array of metabolic and synthetic functions. Indeed, the de Graaf et al. (3) summarize the conundrum of liver function testing by stating that "each quantitative liver function test measures a distinct component of the broader liver function spectrum, which in turn may be differentially influenced during the regenerative process" (3).

In conclusion, the search for the Holy Grail continues, and although

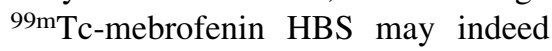

offer the advantage of providing measurement of both uptake and excretory function, the current study serves to illustrate the fact that volumetric, scintigraphic, and metabolic tests may complement one another in providing information on liver function and regeneration at different time points. The Holy Grail may not be a single test, after all, but a combination of complementary assessments.

\section{Pierre M. Gholam}

University Hospitals Case Medical Center Cleveland, Ohio

\section{Zhenghong Lee}

Case Western Reserve University

Cleveland, Ohio

\section{REFERENCES}

1. Tent H, Rook M, Stevens LA, et al. Renal function equations before and after living kidney donation: a within-individual comparison of performance at different levels of renal function. Clin J Am Soc Nephrol. 2010;5:1960-1968.

2. Merlo CA, Weiss ES, Orens JB, et al. Impact of U.S. Lung Allocation Score on survival after lung transplantation. J Heart Lung Transplant. 2009;28: 769-775.

3. de Graaf W, Bennink RJ, Heger M, et al. Quantitative assessment of hepatic function during liver regeneration in a standardized rat model. J Nucl Med. 2010;52:294-302.

4. Sorensen M, Munk OL, Mortensen FV, et al. Hepatic uptake and metabolism of galactose can be quantified in vivo by $2-\left[{ }^{18} \mathrm{~F}\right]$ fluoro-2deoxygalactose positron emission tomography. Am J Physiol Gastrointest Liver Physiol. 2008; 295:G27-G36.

5. Newberry EP, Kennedy SM, Xie Y, et al. Altered hepatic triglyceride content after partial hepatectomy without impaired liver regeneration in multiple murine genetic models. Hepatology. 2008;48:10971105 .

6. Vetelainen R, van Vliet AK, van Gulik TM. Severe steatosis increases hepatocellular injury and impairs liver regeneration in a rat model of partial hepatectomy. Ann Surg. 2007;245:44-50.

7. Palmes D, Spiegel HU. Animal models of liver regeneration. Biomaterials. 2004;25:1601-1611.

8. Sarac TP, Sax HC, Doerr R, Yuksel U, Pulli R, Caruana J. Preoperative fasting improves survival after 90\% hepatectomy. Arch Surg. 1994;129:729733. 